\title{
Estudo de Soluções Aquosas de Copolímeros em Bloco de Poli(Óxido de Etileno)-Poli(Óxido de Propileno) Utilizando Calorimetria Exploratória Diferencial (DSC)
}

\author{
Claudia R. E. Mansur, Gaspar González e Elizabete F. Lucas
}

Resumo: O comportamento de sistemas aquosos contendo copolímeros dibloco monofuncionais de poli(óxido de etileno)-poli(óxido de propileno), bloqueados em uma das extremidades da cadeia com segmento hidrocarbônico linear, foi avaliado através de calorimetria exploratória diferencial (DSC). Transições endotérmicas foram observadas no aquecimento das soluções dos copolímeros e as temperaturas iniciais destas transições estão de acordo com os pontos de turvação dos copolímeros estudados. Nas curvas de resfriamento, são observadas duas transições, e quanto mais rápido é resfriada a solução de copolímero, melhor é observada a transição que deve estar relacionada à formação das micelas. A influência da presença de um agente hidrotrópico também foi avaliada em todas as soluções de copolímeros por meio do aumento no ponto de turvação com o aumento da concentração do agente hidrotrópico, visto que os picos endotérmicos foram deslocados para temperaturas mais altas.

Palavras-chave: Tensoativos, copolímeros, poli(óxido de etileno-b-óxido de propileno), DSC, separação de fases, micelas.

\section{Introdução}

O grupo de tensoativos não iônicos teve seu maior desenvolvimento quando foi descoberto que a variação gradual da hidrofobicidade poderia ser obtida utilizando-se copolímeros em bloco de poli(óxido de etileno) e poli(óxido de propileno) (PEO-PPO), onde a funcionalidade depende do iniciador ${ }^{[1]}$. O poli(óxido de etileno) (PEO) é solúvel em água. O poli(óxido de propileno) (PPO), apesar de possuir estrutura semelhante ao $\mathrm{PEO}$, não é solúvel em água em condições ordinárias, sendo então a parte hidrófoba do tensoativo $^{[2]}$.
A solubilidade dos tensoativos não iônicos em água depende da hidratação dos grupos etoxilados e propoxilados (glicóis) da molécula. Os tensoativos à base de $\mathrm{PEO}$ em solução aquosa apresentam separação de fases com o aumento da temperatura e esta temperatura é conhecida como temperatura ou ponto de turvação ${ }^{[3]}$. Antes de exibirem uma mudança de fases, estes tensoativos podem apresentar solubilidade em água não como unímeros, mas sim sob a forma de agregados moleculares, que são conhecidos como micelas e envolvem essencialmente dois tipos de segmentos: o hidrófobo, que não se mistura bem com o solvente água, e sofre forte atração mú-

Claudia R. E. Mansur e Elizabete F. Lucas, Universidade Federal do Rio de Janeiro / Instituto de Macromoléculas Professora Eloisa Mano (IMA/UFRJ), C. P. 68525, CEP: 21945-970, Rio de Janeiro, RJ, E-mail: elucas@ima.ufrj.br/celias@ima.ufrj.br; Gaspar González, Centro de Pesquisas da Petrobras (CENPES), Ilha do Fundão, Quadra 7. E-mail: gaspar@petrobras.cenpes.com.br. 
tua produzindo uma forma compacta estável; e o hidrófilo, que é muito solúvel e tende a ficar externamente exposto, produzindo uma partícula solúvel $^{[4]}$.

A concentração de tensoativos unímeros, acima da qual há formação de micelas, é chamada de concentração micelar crítica (CMC) e, da mesma forma, a temperatura acima da qual ocorre esta formação é chamada de temperatura micelar crítica (TMC). A formação de micelas não é uma questão de simples separação de fases. Experimentalmente, esta região é determinada pelo plote de medidas de algumas propriedades físicas e espectroscópicas que mudam bruscamente com a formação das micelas como função da concentração do tensoativo ou da temperatura da solução. Tais propriedades compreendem: tensão superficial, densidade, condutância específica, pressão osmótica, detergência, entre outras.

A formação de micelas de copolímeros tribloco difuncionais (HO-PEO-PPO-PEO-OH), conhecidos como PLURONIC ou SYMPERONIC polióis, foi muito estudada devido à importância desses sistemas em diversas aplicações ${ }^{[5-7]}$. Os parâmetros de maior importância estudados foram: a concentração na qual as micelas começam a se formar; fração de moléculas de copolímeros na forma micelar; e o tamanho e o número de agregados das micelas $^{[8-17]}$.

Um estudo termodinâmico de micelização foi desenvolvido envolvendo doze tipos de PLURONIC polióis, possuindo pesos moleculares na faixa de 2.900-14.600 e razões PPO/PEO na faixa de $0,19-1,79^{[12]}$. A formação de micelas é mais difícil em moléculas mais hidrófilas e o PPO exerce maior influência sobre a formação destas micelas do que o PEO. Os PLURONIC polióis de mais alto peso molecular apresentam valores de CMC e TMC mais baixos do que os de mais baixo peso molecular. A entalpia $(\Delta \mathrm{H})$ de micelização é positiva para todos os PLURONIC's analisados, indicando que a transferência dos polímeros em solução para a forma micelar possui uma entalpia desfavorável (processo endotérmico). Foi considerado que a contribuição entrópica $(\Delta S)$ é o fator que favorece o processo de micelização destes copolímeros. Em outro estudo ${ }^{[15]}$, foi observado a transição endotérmica relativa ao processo de micelização destes copolímeros através de análise calorimétrica. Foram calculados os valores desta entalpia de micelização e, também, os valores da TMC, os quais são calculados no início da transição endotérmica. Estes resultados estão em concordância com aqueles obtidos através do estudo anterior ${ }^{[12]}$.

O objetivo deste trabalho é investigar a utilização de uma técnica alternativa para avaliar o comportamento de soluções aquosas de tensoativos. Para tanto, foram estudadas a formação de micelas e a separação de fases de copolímeros em bloco de PEO-PPO em soluções aquosas através de calorimetria exploratória diferencial. Foram avaliados copolímeros dibloco monofuncionais, bloqueados em uma das extremidades de suas cadeias com grupamentos hidrófobos, constituídos de hidrocarbonetos lineares e insaturados, contendo quatro átomos de carbono $\left(\mathrm{C}_{4}\right.$-PEO-PPO-OH e $\mathrm{C}_{4}$-PPO$\mathrm{PEO}-\mathrm{OH})$ ou com misturas de doze e catorze átomos de carbono $\left(\mathrm{C}_{12-14}-\mathrm{PEO}-\mathrm{PPO}-\mathrm{OH}\right.$ e $\mathrm{C}_{12-14}$-PPO-PEO-OH). Além disso, foi estudado também o efeito da presença de um agente hidrotrópico, p-tolueno sulfonato de sódio (NaPTS), no comportamento de fases das soluções aquosas dos copolímeros.

\section{Experimental}

\section{Materiais}

Os copolímeros dibloco monofuncionais de estrutura $\mathrm{C}_{4}$-PEO-PPO-OH e $\mathrm{C}_{4}$-PPO-PEO-OH foram adquiridos da OXITENO (Grupo Ultra-Divisão Química) e os copolímeros $\mathrm{C}_{12-14}$-PEO-PPO-OH e $\mathrm{C}_{12-14}$-PPO-PEO-OH foram adquiridos da Henkel S.A. Indústrias Químicas.

$\mathrm{O}$ agente hidrotrópico, p-tolueno sulfonato de sódio, foi adquirido da COEMA S.A, em solução aquosa a $2,10 \mathrm{M}$.

\section{Secagem dos copolímeros}

Em balão de três bocas e $1000 \mathrm{~mL}$ de capacidade foram colocados $0,05 \mathrm{~mol}$ de copolímero e $400 \mathrm{~mL}$ de tolueno previamente seco. Ao balão, foi adaptado sistema de destilação. O conteúdo do balão foi aquecido em banho de óleo a $120^{\circ} \mathrm{C}$, recolhendo-se aproximadamente $300 \mathrm{~mL}$ de tolueno destilado. O restante de tolueno presente no copolímero foi retirado usando-se evaporador rotatório conectado à bomba de vácuo, à temperatura de $80^{\circ} \mathrm{C}$ e posterior borbulhamento com nitrogênio. 


\section{Caracterização dos copolímeros}

A Tabela 1 mostra a caracterização dos copolímeros dibloco monofuncionais de poli(óxido de etileno)poli(óxido de propileno) (PEO-PPO) ${ }^{[18-20]}$.

\section{Calorimetria exploratória diferencial (DSC)}

As medidas por DSC foram realizadas em calorímetro diferencial de varredura Perkin Elmer DSC7, equipado com o software TAS-7 e acoplado a um computador Perkin Elmer PE-7700. Todas as soluções aquosas dos copolímeros foram analisadas à concentração de $10 \% \mathrm{p} / \mathrm{v}$. As amostras foram estabilizadas durante as medidas e para isso, estas foram deixadas durante 5 minutos nas temperaturas iniciais e finais das análises. Os programas de aquecimento e resfriamento foram variados de $3 \mathrm{a} 15^{\circ} \mathrm{C} / \mathrm{min}$. As cápsulas de alumínio da referência e da amostra foram deixadas sob fluxo de nitrogênio $\left(30 \mathrm{~cm}^{3} / \mathrm{min}\right)$ durante as medidas para prevenir a formação de bolhas e/ou evaporação do solvente a mais altas temperaturas. As calibrações da temperatura de fusão $\left(\mathrm{T}_{\mathrm{m}}\right)$ e do calor de fusão $\left(\Delta \mathrm{H}_{\mathrm{m}}\right)$ foram feitas utilizando-se como padrão o elemento químico índio (In). Esta amostra padrão foi avaliada nas mesmas taxas de aquecimento utilizadas para as amostras $\left(3-10^{\circ} \mathrm{C} /\right.$ min) e foi observado que os valores da $\mathrm{T}_{\mathrm{m}}$ e $\Delta \mathrm{H}_{\mathrm{m}}$ não sofreram variação significativa, apresentando $\mathrm{T}_{\mathrm{m}}=155,9 \pm 0,6^{\circ} \mathrm{C}$ e $\Delta \mathrm{H}_{\mathrm{m}}=28,5 \pm 0,1 \mathrm{~J} / \mathrm{g}$. Todos os valores de $\Delta \mathrm{H}$ obtidos para as soluções dos copolímeros analisadas foram corrigidos com os valores da constante do equipamento ( $\mathrm{K}$ em torno de 1 ), calculada através da Equação 1.

$$
\mathrm{K}=\frac{\mathrm{m}_{\text {In }}}{\mathrm{A}} \mathrm{x} \Delta \mathrm{H}_{\text {In }}
$$

Onde,

$\mathrm{m}_{\text {In }}=$ massa do índio utilizada na análise;

$\mathrm{A}$ = área do pico endotérmico obtido na análise do índio;

$\Delta \mathrm{H}_{\mathrm{In}}=$ variação de entalpia teórica do índio.

As temperaturas iniciais e finais das transições endotérmicas e exotérmicas, medidas por DSC, foram obtidas na temperatura na qual o prolongamento da linha base não acompanha o pico.

\section{Resultados e Discussão}

\section{Soluções aquosas de copolímeros dibloco monofuncionais PEO-PPO}

Estudo da formação de micelas e separação de fases através de medidas de tensão superficial e método visual, respectivamente

O estudo da formação de micelas e separação de fases das soluções aquosas de copolímeros dibloco à base de PEO-PPO bloqueados com grupamentos $\mathrm{C}_{4}$ e $\mathrm{C}_{12-14}$ vem sendo realizado em nosso laboratório de pesquisas $^{[19-21]}$. A formação de micelas nestes sistemas tem sido avaliada através da medida da tensão superficial em função da concentração, o que permite determinar as concentrações e temperaturas micelares críticas (CMC e TMC, respectivamente) ${ }^{[20,21]}$. Já o estudo da separação de fases tem sido

Tabela 1. Caracterização dos copolímeros dibloco mono-funcionais de poli(óxido de etileno)-poli(óxido de propileno) ${ }^{[18-20]}$

\begin{tabular}{|c|c|c|c|c|c|c|c|}
\hline \multirow{2}{*}{$\begin{array}{l}\text { Estrutura do } \\
\text { copolímero }\end{array}$} & \multirow[t]{2}{*}{$\overline{\mathbf{M}}_{\mathbf{n}}^{(a)}$} & \multirow[t]{2}{*}{$\overline{\mathbf{M}}_{w} / \bar{M}_{n}^{(b)}$} & \multicolumn{2}{|c|}{$\begin{array}{l}\text { Unidades de }{ }^{(c)} \\
\text { monômero repetidas }\end{array}$} & \multirow[t]{2}{*}{$\begin{array}{c}\text { Razão } \\
\text { EO/PO }\end{array}$} & \multirow[t]{2}{*}{$\begin{array}{l}\text { TMC } \\
\left({ }^{\circ} \mathbf{C}\right)^{(\mathbf{d})}\end{array}$} & \multirow{2}{*}{$\begin{array}{l}\text { Ponto de } \\
\text { turvação } \\
\left({ }^{\circ} \mathrm{C}\right)^{(\mathrm{e})}\end{array}$} \\
\hline & & & EO & PO & & & \\
\hline $\mathrm{C}_{4}-(\mathrm{EO})_{4}-(\mathrm{PO})_{11}-\mathrm{OH}$ & 920 & 1,24 & 4 & 11 & 0,36 & - & 33,0 \\
\hline $\mathrm{C}_{4}-(\mathrm{PO})_{10}-(\mathrm{EO})_{6}-\mathrm{OH}$ & 900 & 1,23 & 6 & 10 & 0,60 & 16,0 & 28,0 \\
\hline $\mathrm{C}_{12-14}-(\mathrm{PO})_{4}-(\mathrm{EO})_{5}-\mathrm{OH}$ & 600 & 1,15 & 5 & 4 & 1,25 & - & 13,0 \\
\hline $\mathrm{C}_{12-14}-(\mathrm{EO})_{6}-(\mathrm{PO})_{5}-\mathrm{OH}$ & 720 & 1,15 & 6 & 5 & 1,20 & 9,0 & 25,0 \\
\hline $\mathrm{C}_{12-14}-(\mathrm{EO})_{9}-(\mathrm{PO})_{6}-\mathrm{OH}$ & 910 & 1,19 & 9 & 6 & 1,50 & 12,0 & 39,0 \\
\hline
\end{tabular}

(a) Determinado através da osmometria de pressão de vapor (VPO);

(b) Determinado através da cromatografia de permeação em gel (GPC);

(c) Determinado através da espectroscopia de ressonância magnética nuclear de hidrogênio $\left({ }^{1} \mathrm{H}-\mathrm{NMR}\right)$;

(d) Determinado através da medida da tensão superficial em função da concentração das soluções aquosas dos copolímeros de PEO-PPO. Concentração $=10 \% \mathrm{p} / \mathrm{v}$;

(e) Determinado através da construção do diagrama de fases temperatura versus concentração das soluções aquosas dos copolímeros de PEO-PPO. Concentração $=10 \% \mathrm{p} / \mathrm{v}$. 
desenvolvido através da construção de diagramas de fases temperatura versus concentração, determinando o ponto de turvação dos sistemas visualmente ${ }^{[19]}$. A Tabela 1 mostra os resultados obtidos através dos métodos citados acima para soluções aquosas de copolímeros dibloco monofuncionais de PEO-PPO a uma concentração de copolímero de $10 \% \mathrm{p} / \mathrm{v}$.

$\mathrm{O}$ copolímero em bloco de estrutura $\mathrm{C}_{4^{-}}(\mathrm{PO})_{10^{-}}$ (EO) $)_{6}-\mathrm{OH}$ apresenta pontos de turvação mais baixos do que o copolímero em bloco de $\mathrm{C}_{4}-(\mathrm{EO})_{4}-(\mathrm{PO})_{11}-\mathrm{OH}$, apesar do primeiro possuir maior razão EO/PO (Tabela 1). Este fato foi atribuido à posição dos segmentos hidrófilos $(\mathrm{EO}$ e $\mathrm{OH})$ e hidrófobos $\left(\mathrm{C}_{4}\right.$ e $\left.\mathrm{PO}\right)$ nestes copolímeros. Ao se comparar resultados dos copolímeros bloqueados com o grupamento $\mathrm{C}_{12-14}$, como esperado, observa-se que aquele que possui maior razão $\mathrm{EO} / \mathrm{PO}$, $\mathrm{C}_{12-14}-(\mathrm{EO})_{9}-(\mathrm{PO})_{6}-\mathrm{OH}$ apresenta os maiores pontos de turvação, e os outros dois copolímeros apresentam pontos de turvação diferentes, apesar de possuirem razões $\mathrm{EO} / \mathrm{PO}$ semelhantes (Tabela 1). $\mathrm{O}$ copolímero $\mathrm{C}_{12-14^{-}}$ (EO) $)_{6}-(\mathrm{PO})_{5}-\mathrm{OH}$, que possui grupos hidrófilos (EO e $\mathrm{OH})$ e hidrófobos $\left(\mathrm{C}_{12-14} \mathrm{e} \mathrm{PO}\right)$ em posições alternadas, apresenta maiores pontos de turvação do que o copolímero $\mathrm{C}_{12-14}-(\mathrm{PO})_{4}-(\mathrm{EO})_{5}-\mathrm{OH}$, que possui os segmentos hidrófilos/hidrófobos em posições adjacentes. Este comportamento é semelhante ao observado para os copolímeros cujo bloqueio é $\mathrm{C}_{4}$. Foi sugerido, neste caso, que a estrutura do copolímero apresenta uma forte influência sobre sua solubilidade em água. Além disso, o comportamento apresentado por estes copolímeros em solução aquosa foi atribuído à facilidade estrutural de associação molecular observada nos copolímeros que possuem segmentos hidrófilos/ hidrófobos adjacentes, como mostrado no modelo proposto em estudos prévios ${ }^{[19]}$.

Copolímeros bloqueados com o grupamento $\mathrm{C}_{12-14}$ apresentam valores mais baixos de TMC do que os encontrados para os copolímeros bloqueados com o grupamento $\mathrm{C}_{4}$. Este comportamento também é observado quando se analisa a solubilidade destes copolímeros: quanto maior o bloqueio, menor o ponto de turvação, e isso foi atribuído ao maior segmento hidrófobo presente nos copolímeros bloqueados com $\mathrm{C}_{12-14}$. Os valores de TMC para os copolímeros $\mathrm{C}_{4}-(\mathrm{EO})_{4}-(\mathrm{PO})_{11}-\mathrm{OH}$ e $\mathrm{C}_{12-14^{-}}(\mathrm{PO})_{4}-(\mathrm{EO})_{5}-\mathrm{OH}$ não são detectados dentro da faixa de temperatura de trabalho $\left(8-40^{\circ} \mathrm{C}\right)^{[20]}$. O primeiro, por ser o mais solúvel e, também, apresentar valor de CMC alto, e o segundo, por razão contrária, por ser o menos solúvel e apresentar valor de CMC muito baixo.
Estudo da influência das taxas de aquecimento e resfriamento nas curvas de DSC

Um estudo preliminar variando-se as taxas de aquecimento e resfriamento teve como finalidade escolher as melhores condições para os estudos por DSC das soluções aquosas dos copolímeros, bem como observar sua influência sobre a separação de fases e a formação de micelas nas soluções estudadas.

As Figuras 1a, 1b e 1c mostram os resultados obtidos por DSC da água e das soluções aquosas do copolímero $\mathrm{C}_{12-14}-(\mathrm{EO})_{6}-(\mathrm{PO})_{5}-\mathrm{OH}$ a diferentes taxas de aquecimento e resfriamento: 5,$0 ; 7,5$ e $10^{\circ} \mathrm{C} / \mathrm{min}$, res-
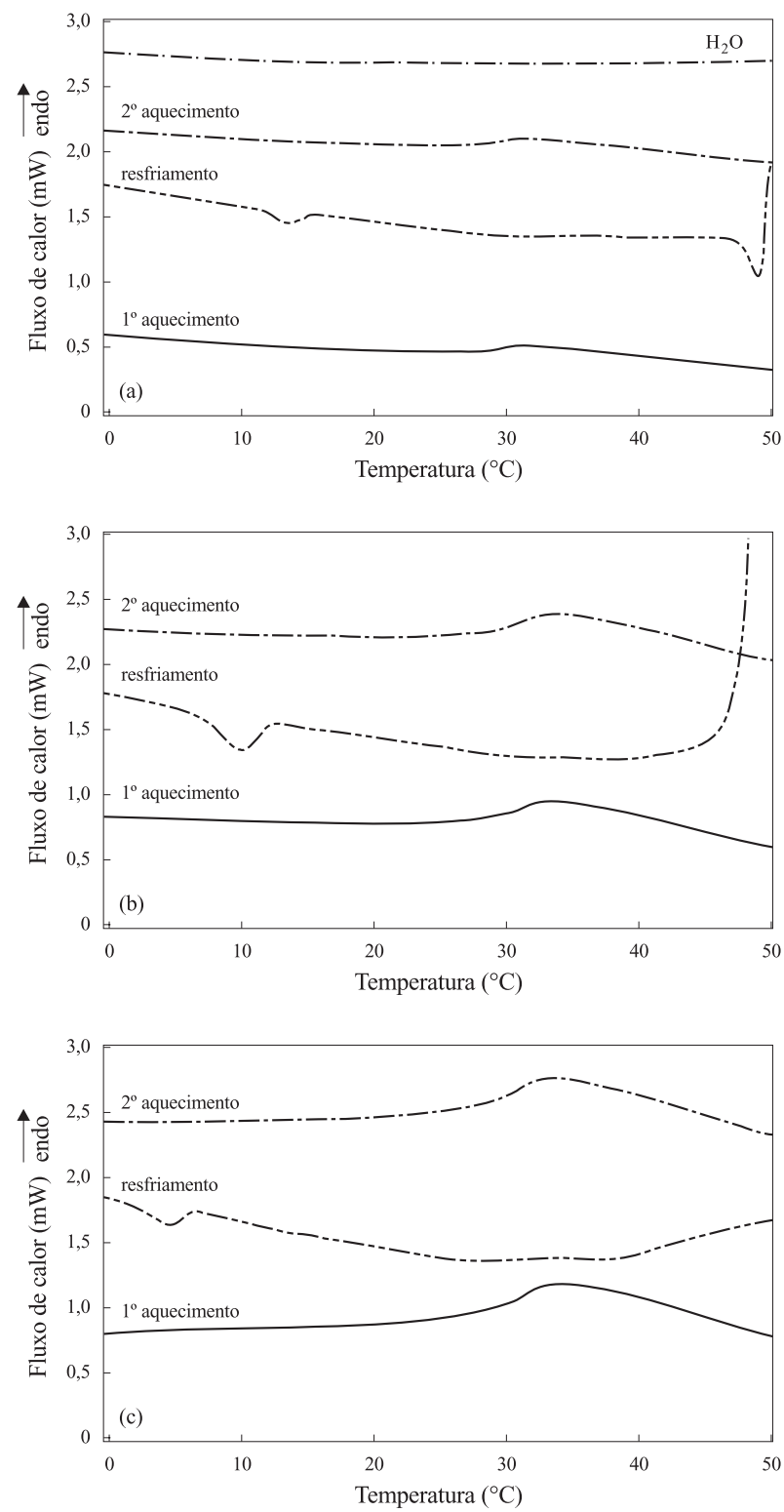

Figura 1. Curvas DSC das soluções aquosas do copolímero $\mathrm{C}_{12-14}$-(EO) $)_{6}-(\mathrm{PO})_{5}-\mathrm{OH}, 10 \% \mathrm{p} / \mathrm{v}$, a diferentes taxas de aquecimento e resfriamento: (a) $5^{\circ} \mathrm{C} / \mathrm{min}$; (b) $7,5^{\circ} \mathrm{C} / \mathrm{min}$; (c) $10^{\circ} \mathrm{C} / \mathrm{min}$. 

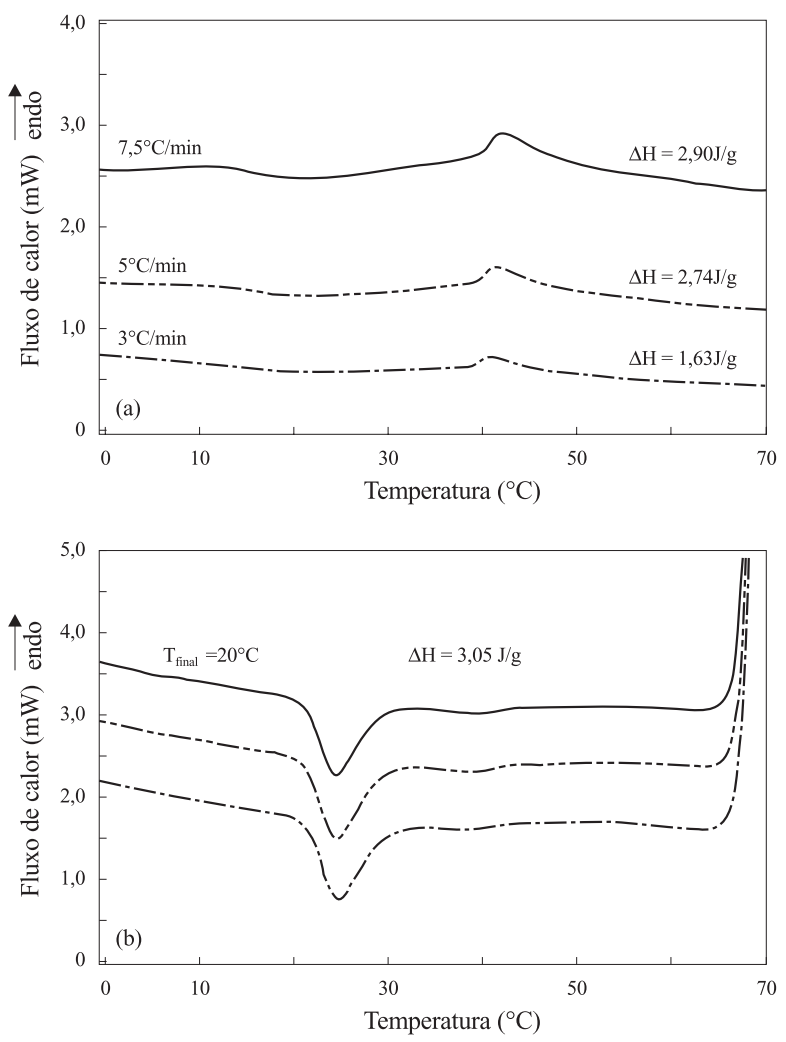

Figura 2. Curvas DSC da solução aquosa do copolímero $\mathrm{C}_{12-14}-(\mathrm{EO})_{9}-(\mathrm{PO})_{6}-\mathrm{OH}, 10 \% \mathrm{p} / \mathrm{v}$ : (a) a diferentes taxas de aquecimento e (b) resfriamento constante a $7,5^{\circ} \mathrm{C} / \mathrm{min}$.

pectivamente. No aquecimento de cada solução foi observado um pico endotérmico, o qual não foi observado quando se analisou a água pura. Para todas as soluções a temperatura inicial foi de aproximadamente $26^{\circ} \mathrm{C}$. Uma vez que a separação de fases do copolímero analisado em solução aquosa de $10 \% \mathrm{p} / \mathrm{v}$ ocorre à temperatura de $25^{\circ} \mathrm{C}$ (Tabela 1$)^{[19]}$, tal transição endotérmica foi atribuída a este processo de separação de fases. A temperatura inicial do pico endotérmico não apresentou variação significativa do primeiro para o segundo aquecimento. Tendo em vista que as variações observadas foram as mesmas apresentadas pelo padrão (índio), pode-se então considerar tal processo neste sistema como reversível. No resfriamento, o pico exotérmico, que corresponderia ao processo inverso de separação de fases, não ficou bem definido nestas condições de análise. Porém, foi observado um segundo pico exotérmico, o qual pode ser atribuído ao processo de hidratação dos grupos PO. Quanto ao valor da temperatura final desta transição, pode-se observar que este varia de acordo com as taxas de resfriamento utilizadas.

Tendo em vista que as variações observadas foram as mesmas apresentadas pelo padrão (índio), foi feito um estudo observando-se o comportamento dos sistemas durante o resfriamento, mantendo-se constante as taxas de resfriamento e variando-se as taxas de aquecimento da solução aquosa do copolímero $\mathrm{C}_{12-14^{-}}(\mathrm{EO})_{9^{-}}$ $(\mathrm{PO})_{6}-\mathrm{OH}$. A Figura 2a mostra o aquecimento da solução de copolímero e é observada uma variação no valor de $\Delta \mathrm{H}$ do pico endotérmico, de acordo com a taxa de aquecimento usada, mas a temperatura inicial desta transição endotérmica foi concordante com o ponto de turvação determinado previamente (Tabela 1) ${ }^{[19]}$. Já a Figura $2 \mathrm{~b}$ mostra que as temperaturas iniciais e os valores de $\Delta \mathrm{H}$ das transições exotérmicas mais pronunciadas observadas no resfriamento não sofreram variação significativa (a exemplo do observado na Figura 1a). Porém a temperatura final da transição, a qual seria esperado corresponder ao processo de hidratação dos grupos PO, não está de acordo com a TMC calculada a partir do método discutido anteriormente ${ }^{[20]}$. Este resultado indica que medidas realizadas à taxa de resfriamento constante, igual a $7,5^{\circ} \mathrm{C} / \mathrm{min}$ (independente da taxa de aquecimento utilizada), não são adequadas para obten-

Tabela 2. Resultados obtidos por DSC das soluções aquosas do copolímero $\mathrm{C}_{12-14}-(\mathrm{EO})_{9}-(\mathrm{PO})_{6}-\mathrm{OH}$

\begin{tabular}{|c|c|c|c|c|c|c|}
\hline \multirow{2}{*}{$\begin{array}{l}\text { Taxa de resfriamento } \\
\left({ }^{\circ} \mathrm{C} / \mathrm{min}\right)\end{array}$} & \multicolumn{2}{|c|}{ Aquecimento $5^{\circ} \mathrm{C} / \mathrm{min}^{(\text {a) }}$} & \multicolumn{2}{|c|}{ Aquecimento $7,5^{\circ} \mathrm{C} / \mathrm{min}^{(\mathrm{d})}$} & \multicolumn{2}{|c|}{ Aquecimento $10^{\circ} \mathrm{C} / \mathrm{min}^{(\mathrm{e})}$} \\
\hline & $\mathbf{T}_{\mathbf{f}}\left({ }^{\circ} \mathbf{C}\right)^{(\mathbf{b})}$ & $\Delta \mathbf{H}(\mathbf{J} / \mathbf{g})^{(\mathrm{C})}$ & $\mathbf{T}_{\mathrm{f}}\left({ }^{\circ} \mathbf{C}\right)^{(\mathbf{b})}$ & $\Delta \mathbf{H}(\mathbf{J} / \mathbf{g})^{(\mathrm{C})}$ & $\mathbf{T}_{\mathbf{f}}\left({ }^{\circ} \mathbf{C}\right)^{(\mathbf{b})}$ & $\Delta \mathbf{H}(\mathbf{J} / \mathbf{g})^{(\mathrm{C})}$ \\
\hline 3,0 & 26,6 & $-1,7$ & 26,8 & $-1,7$ & 27,2 & $-1,8$ \\
\hline 5,0 & 23,4 & $-2,4$ & 24,1 & $-1,5$ & 24,7 & $-1,7$ \\
\hline 7,5 & 21,1 & $-2,5$ & 20,5 & $-1,6$ & 20,9 & $-1,7$ \\
\hline 10,0 & 16,7 & $-2,5$ & 17,0 & -16 & 17,7 & $-1,7$ \\
\hline 15,0 & - & - & - & - & 12,3 & $-1,7$ \\
\hline
\end{tabular}

(a) Temperatura inicial da transição endotérmica obtida no primeiro aquecimento $=40^{\circ} \mathrm{C} ; \quad \Delta \mathrm{H}=2,524 \mathrm{~J} / \mathrm{g}$;

(b) Temperaturas finais da segunda transição exotérmica obtida no resfriamento;

(c) $\Delta \mathrm{H}$ da segunda transição exotérmica obtida no resfriamento;

(d) Temperatura inicial da transição endotérmica obtida no primeiro aquecimento $=39^{\circ} \mathrm{C} ; \quad \Delta \mathrm{H}=2,779 \mathrm{~J} / \mathrm{g}$;

(e) Temperatura inicial da transição endotérmica obtida no primeiro aquecimento $=39^{\circ} \mathrm{C} ; \quad \Delta \mathrm{H}=3,027 \mathrm{~J} / \mathrm{g}$ 

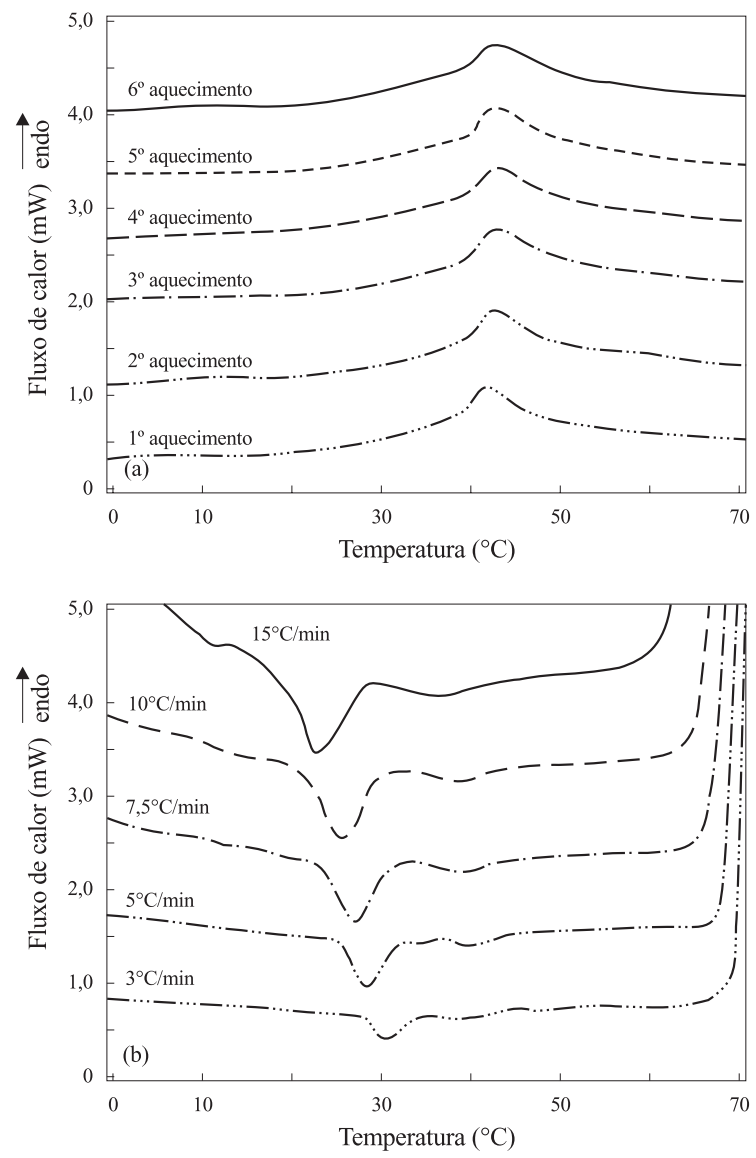

Figura 3. Curvas DSC da solução aquosa do copolímero $\mathrm{C}_{12-14^{-}}-(\mathrm{EO})_{9}-(\mathrm{PO})_{6}-\mathrm{OH}, 10 \% \mathrm{p} / \mathrm{v}$ : (a) aquecimento a $10^{\circ} \mathrm{C} / \mathrm{min}$ e (b) resfriamento de 3 a $15^{\circ} \mathrm{C} / \mathrm{min}$.

ção de resultados comparáveis àqueles já determinados por outros métodos ${ }^{[19-21]}$.

A solução aquosa do copolímero $\mathrm{C}_{12-14^{-}}(\mathrm{EO})_{9^{-}}$ $(\mathrm{PO})_{6}-\mathrm{OH}$ foi então avaliada a diferentes taxas de resfriamento. A Figura 3 mostra as curvas de DSC das medidas realizadas à taxa de aquecimento constante, a $10^{\circ} \mathrm{C} / \mathrm{min}$, e variando-se as taxas de resfriamento $(3 ; 5$; 7,$5 ; 10$ e $\left.15^{\circ} \mathrm{C} / \mathrm{min}\right)$. Duas transições foram observadas durante o resfriamento da solução de copolímero (Fi- gura 3b). Uma delas está relacionada ao inverso do ponto de turvação dos sistemas (primeiro pico exotérmico) e a outra, está relacionada ao inverso do processo no qual ocorre o rompimento das interações água-PO (segundo pico exotérmico). Três conjuntos de resultados foram obtidos para cada taxa de aquecimento $\left(5 ; 7,5\right.$ e $10^{\circ} \mathrm{C} /$ $\mathrm{min}$ ) variando-se a taxa de resfriamento. A temperatura final e $\Delta H$ do segundo pico exotérmico observado em cada taxa de resfriamento são mostrados na Tabela 2 . Como esperado, todos os valores de temperatura final variaram com a taxa de resfriamento e não sofreram variação significativa com a mudança da taxa de aquecimento. Porém, a taxa de aquecimento de $10^{\circ} \mathrm{C} / \mathrm{min}$ foi escolhida para a realização das medidas posteriores, uma vez que ainda foi possível visualizar o pico de interesse quando a medida foi realizada a taxa de resfriamento de $15^{\circ} \mathrm{C} / \mathrm{min}$ (Figura $3 \mathrm{~b}$ ).

\section{Estudo do processo de separação de fases e com-} portamento de micelas por DSC

As medidas das soluções aquosas dos copolímeros de PEO-PPO foram realizadas utilizando-se taxa de aquecimento constante e igual a $10^{\circ} \mathrm{C} / \mathrm{min}$ (Figura 3a) e taxas de resfriamento de 3 a $15^{\circ} \mathrm{C} / \mathrm{min}$ (Figura $3 b$ ). Os resultados obtidos para todos os copolímeros analisados são mostrados na Tabela 3. As variações de entalpia da transição térmica observada no aquecimento para os vários copolímeros são baixas e todos os copolímeros analisados exibiram valores de entalpia em torno de 3 a $4 \mathrm{~J} / \mathrm{g}$, a exceção do copolímero $\mathrm{C}_{12-14}-(\mathrm{PO})_{4}-(\mathrm{EO})_{5}-\mathrm{OH}$. Os valores de $\Delta \mathrm{H}$ não apresentaram variação significativa em função das condições de análise.

Como já descrito anteriormente, duas transições foram observadas durante o resfriamento das soluções (Figura 3b). Os copolímeros que são bloqueados com grupamento menor $\left(\mathrm{C}_{4}\right)$ apresentam picos exotérmicos

Tabela 3. Resultados obtidos por DSC das soluções aquosas do copolímeros de PEO-PPO (a)

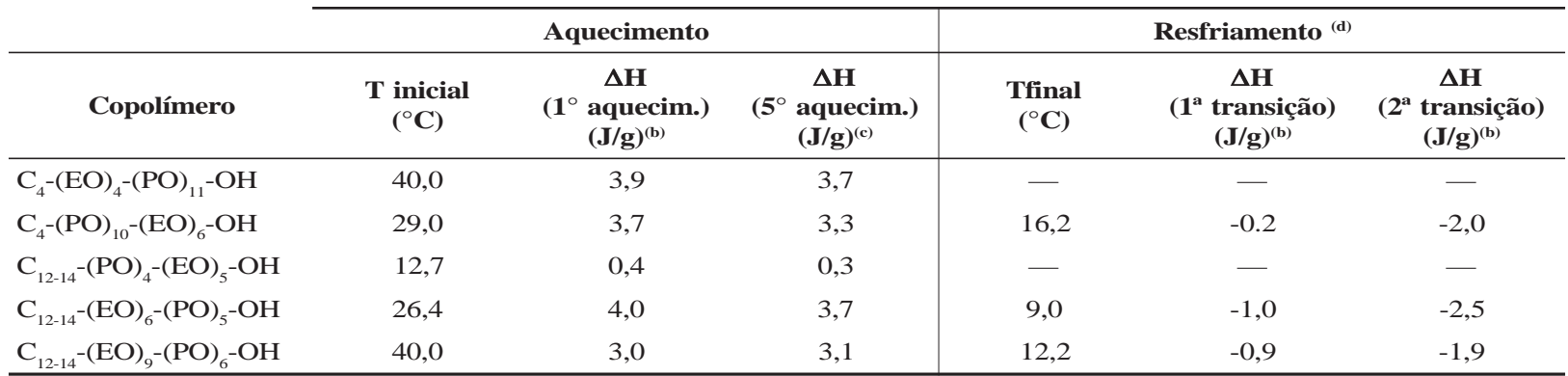

(a) Valores obtidos a taxa de aquecimento contante em $10^{\circ} \mathrm{C} / \mathrm{min}$;

(b) Medida realizada durante o primeiro aquecimento da solução polimérica;

${ }^{\text {(c) }}$ Medida realizada após o processo de resfriamento a taxa de $10^{\circ} \mathrm{C} / \mathrm{min}$;

(d) Valores obtidos no processo de resfriamento a taxa de $10^{\circ} \mathrm{C} / \mathrm{min}$. 


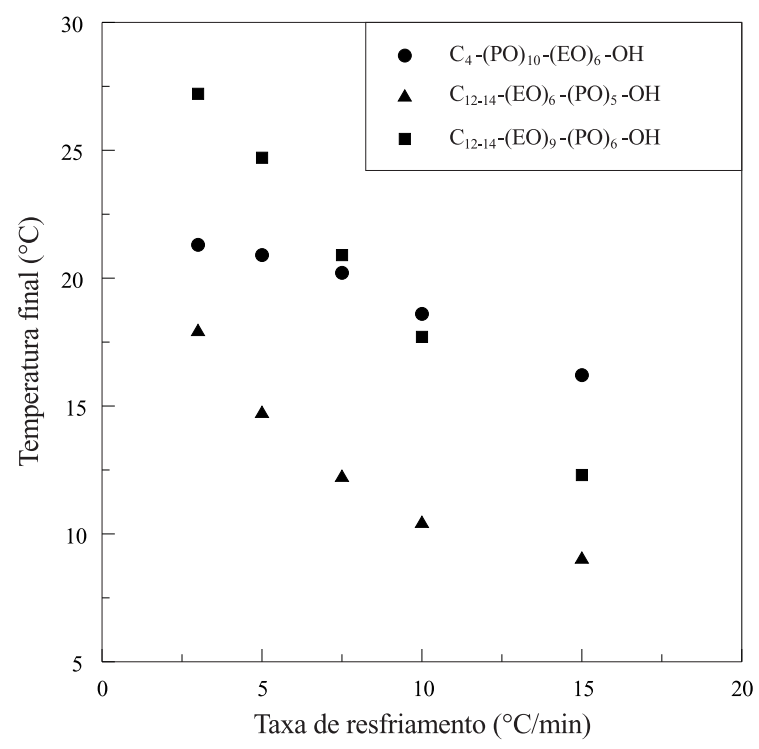

Figura 4. Temperatura final do segundo pico observado durante o resfriamento das soluções aquosas dos copolímeros em função da taxa de resfriamento utilizada.

com pior definição. Os resultados para os copolímeros $\mathrm{C}_{4}$-(EO) $)_{4}-(\mathrm{PO})_{11}-\mathrm{OH}$ e $\mathrm{C}_{12-14}-(\mathrm{PO})_{4}-(\mathrm{EO})_{5}-\mathrm{OH}$ não são apresentados por não ter sido possível obter informações no resfriamento das soluções destes copolímeros. Comparando os valores de $\Delta \mathrm{H}$ das transições obtidas no aquecimento e no resfriamento, pode-se observar que o valor de $\Delta \mathrm{H}$ da transição obtida no aquecimento é similar à soma dos valores de $\Delta \mathrm{H}$ das transições obtidas no resfriamento. Este comportamento não foi observado para o copolímero $\mathrm{C}_{4}-(\mathrm{PO})_{10}-(\mathrm{EO})_{6}-\mathrm{OH}$ que, devido à pouca definição das transições exotérmicas, pode ter acarretado erros significativos nos cálculos. Isto sugere que a transição observada no aquecimento deve corresponder aos fenômenos de desidratação dos grupos PO e separação de fases, os quais não foram resolvidos em duas transições distintas. De acordo com esta discussão, a explicação para o fato do copolímero $\mathrm{C}_{12-14}-(\mathrm{PO})_{4}-(\mathrm{EO})_{5}-\mathrm{OH}$ apresentar valor de $\Delta \mathrm{H}$ (da transição observada no aquecimento) muito pequeno reside no fato de que somente está ocorrendo o processo de separação de fases na faixa de temperatura utilizada. Durante o resfriamento não são observados os dois picos relativos aos processos inversos de desidratação dos grupos PO e separação de fases. Tal discussão está coerente com os resultados anteriores obtidos por outras técnicas ${ }^{[19-21]}$, que mostram uma CMC muito baixa e uma TMC a qual não foi possível de ser medida. Conforme a taxa de resfriamento é aumentada, as transições relacionadas ao processo inverso de desidratação dos grupos PO tornam-se mais evidentes; além disso, são deslocadas para mais baixas temperaturas (o que não ocorre com a transição relacionada ao processo inverso de separação de fases). Todos os copolímeros analisados apresentaram o mesmo comportamento, logo foi construído um gráfico da temperatura final, observada na segunda transição exotérmica, como função da taxa de resfriamento (Figura 4). Foi observado que aumentando a taxa de resfriamento, a temperatura diminui e tende a ficar constante, em torno dos valores obtidos de TMC por outros métodos ${ }^{[20]}$ (Tabela 1). Acima de $15^{\circ} \mathrm{C} / \mathrm{min}$, as transições exotérmicas obtidas no resfriamento não foram bem definidas, devido a limitações do instrumento utilizado.

\section{Soluções aquosas de copolímeros dibloco monofuncionais PEO-PPO contendo um agente hidrotrópico}

\section{Estudo da separação de fases e agregação por DSC}

A calorimetria exploratória diferencial foi também usada para estudar as soluções aquosas dos copolímeros dibloco monofuncionais de PEO-PPO contendo um agente hidrotrópico, o p-tolueno sulfonato de sódio (NaPTS).

$\mathrm{Na}$ literatura ${ }^{[22]}$ tem sido mostrado que hidrótropos ou agentes hidrotrópicos são compostos orgânicos que aumentam a solubilidade em água de compostos orgânicos pouco solúveis. $\mathrm{O}$ mecanismo molecular da solubilização hidrotrópica não foi esclarecido ainda, porém parece claro que a hidrotropia difere da solubilização micelar e do efeito "saltingin", promovido por alguns sais inorgânicos.

Através de estudo realizado anteriormente ${ }^{[20]}$, foi observado que o NaPTS pode também apresentar agregados em solução aquosa, e a formação destes também depende da temperatura e/ou concentração. A concentração na qual se observou esta agregação, a $30^{\circ} \mathrm{C}$, foi de aproximadamente $0,5 \mathrm{M}$.

Os resultados de DSC das soluções aquosas de NaPTS foram obtidos nas mesmas condições utilizadas no estudo das soluções aquosas dos copolímeros de PEOPPO. A Figura 5 mostra as curvas DSC do primeiro aquecimento, o resfriamento a $10^{\circ} \mathrm{C} / \mathrm{min}$ e do último aquecimento da solução aquosa de 2,10 M de NaPTS. Um pico endotérmico foi observado durante o primeiro aquecimento, e sua temperatura inicial é similar ao ponto de agregação do $\mathrm{NaPTS}\left(\sim 30^{\circ} \mathrm{C}\right)$, visto que a solução aquosa de NaPTS não apresentou separação de fases 


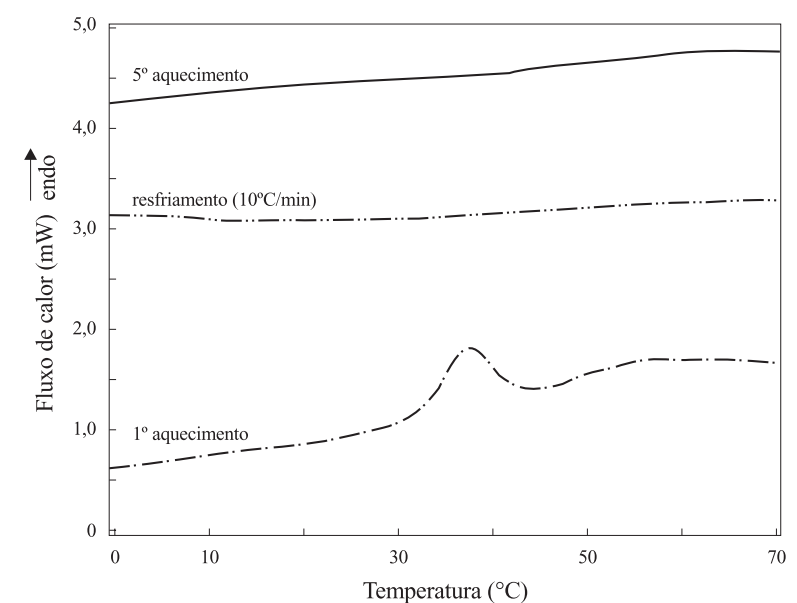

Figura 5. Curvas DSC da solução aquosa de NaPTS, a 2,10M, durante o primeiro e o último aquecimento e o resfriamento.

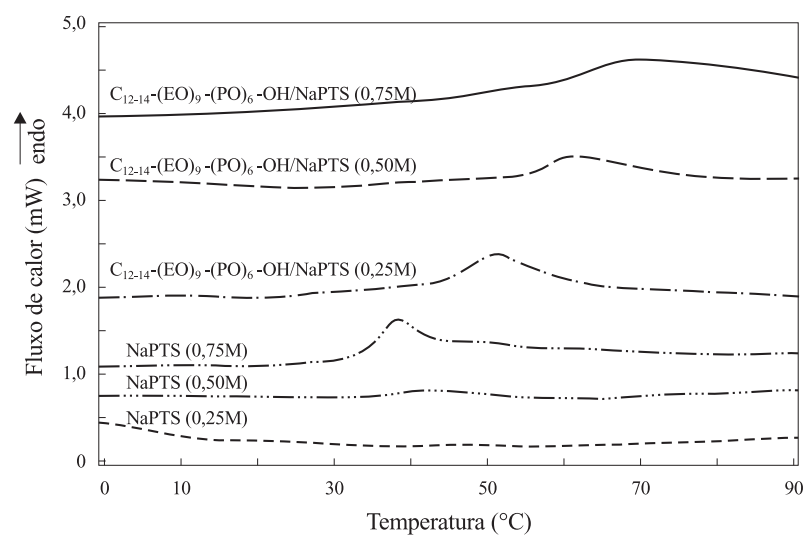

Figura 6. Curvas DSC das soluções aquosas de NaPTS, a 0,$25 ; 0,50$ e $0,75 \mathrm{M}$, durante o primeiro aquecimento, com e sem a adição do copolímero $\mathrm{C}_{12-14}-(\mathrm{EO})_{9}-(\mathrm{PO})_{6}-\mathrm{OH}$, a $10 \% \mathrm{p} / \mathrm{v}$

quando foi analisada pelo método visual ${ }^{[19]}$. Não foi observada nenhuma transição no resfriamento, em todas as taxas utilizadas $\left(3-10^{\circ} \mathrm{C} / \mathrm{min}\right)$, nem nos demais aquecimentos. Logo, a agregação do NaPTS pode ser um processo irreversível ou as condições de análise utilizadas não foram adequadas para ser observada a transição relacionada a este processo.

As medidas das soluções aquosas dos copolímeros de PEO-PPO contendo NaPTS foram conduzidas nas mesmas condições anteriores. A Figura 6 mostra as curvas DSC dos primeiros aquecimentos para as soluções aquosas de NaPTS a 0,25; 0,50 e $0,75 \mathrm{M}$ e as soluções aquosas do copolímero $\mathrm{C}_{12-14^{-}}(\mathrm{EO})_{9}-(\mathrm{PO})_{6}$ $\mathrm{OH}(\mathrm{a} 10 \% \mathrm{p} / \mathrm{v})$ contendo NaPTS nas mesmas concentrações. As soluções de NaPTS apresentaram comportamento semelhante ao observado na Figura 5, exceto para a solução de NaPTS a $0,25 \mathrm{M}$, que não apresentou a formação de agregados na faixa de temperatura utilizada. O início destas transições nas soluções aquosas de NaPTS a 0,50 e $0,75 \mathrm{M}$ foi observado na mesma temperatura que para a solução a
$2,10 \mathrm{M}$, em torno de $30^{\circ} \mathrm{C}$ (os valores de temperatura de início da transição foram tomados de curvas expandidas). Analisando-se as soluções dos copolímeros contendo NaPTS, foram observados picos endotérmicos de um processo reversível, relativos aos pontos de turvação das soluções e os resultados são mostrados na Tabela 4. O efeito hidrotrópico foi observado para todos os copolímeros, devido aos picos endotérmicos terem sido deslocados para temperaturas mais altas, ou seja, os pontos de turvação aumentaram com o aumento da concentração de NaPTS. A

Tabela 4. Resultados obtidos por DSC das soluções aquosas do copolímeros de PEO-PPO contendo NaPTS

\begin{tabular}{|c|c|c|}
\hline Amostra & $\mathbf{T}$ inicial $\left({ }^{\circ} \mathbf{C}\right){ }^{(a)}$ & $\Delta \mathbf{H}(\mathbf{J} / \mathbf{g})^{(\mathbf{b})}$ \\
\hline \multicolumn{3}{|c|}{$\mathrm{C}_{4}-(\mathrm{EO})_{4}-(\mathrm{PO})_{11}-\mathrm{OH}$} \\
\hline $0,25 \mathrm{M}$ & 42 & 2,2 \\
\hline $0,50 \mathrm{M}$ & 50 & 2,1 \\
\hline $0,75 \mathrm{M}$ & 55 & 1,3 \\
\hline \multicolumn{3}{|c|}{$\mathrm{C}_{4}-(\mathrm{PO})_{10}-(\mathrm{EO})_{6}-\mathrm{OH}$} \\
\hline $0,25 \mathrm{M}$ & 31 & 2,0 \\
\hline $0,50 \mathrm{M}$ & 35 & 1,9 \\
\hline $0,75 \mathrm{M}$ & 39 & 1,2 \\
\hline \multicolumn{3}{|c|}{$\mathrm{C}_{12-14}-(\mathrm{PO})_{4}-(\mathrm{EO})_{5}-\mathrm{OH}$} \\
\hline $0,25 \mathrm{M}$ & 22 & 0,4 \\
\hline $0,50 \mathrm{M}$ & 30 & 0,3 \\
\hline $0,75 \mathrm{M}$ & 40 & 0,2 \\
\hline \multicolumn{3}{|c|}{$\mathrm{C}_{12-14}-(\mathrm{EO})_{6}-(\mathrm{PO})_{5}-\mathrm{OH}$} \\
\hline $0,25 \mathrm{M}$ & 30 & 3,3 \\
\hline $0,50 \mathrm{M}$ & 38 & 3,1 \\
\hline $0,75 \mathrm{M}$ & 40 & 3,0 \\
\hline \multicolumn{3}{|c|}{$\mathrm{C}_{12-14}-(\mathrm{EO})_{9}-(\mathrm{PO})_{6}-\mathrm{OH}$} \\
\hline $0,25 \mathrm{M}$ & 48 & 2,3 \\
\hline $0,50 \mathrm{M}$ & 56 & 1,9 \\
\hline $0,75 \mathrm{M}$ & 60 & 1,4 \\
\hline \multicolumn{3}{|l|}{ NaPTS } \\
\hline $0,25 \mathrm{M}$ & - & - \\
\hline $0,50 \mathrm{M}$ & 30 & 0,1 \\
\hline $0,75 \mathrm{M}$ & 30 & 0,2 \\
\hline $2,10 \mathrm{M}$ & 30 & 0,8 \\
\hline
\end{tabular}

(a) Valores obtidos no início da transição endotérmica do processo de aquecimento após processo de resfriamento de $10^{\circ} \mathrm{C} / \mathrm{min}$.

(b) Valores obtidos no primeiro aquecimento das soluções. 
transição relativa à agregação de NaPTS não foi observada nestas análises.

Através dos resultados obtidos pode-se concluir que, durante o aquecimento, o processo de desidratação dos grupos PO e separação de fases ocorrem, apesar de não exibirem transições distintas. O comportamento das curvas das misturas de NaPTS/copolímero sugere que ocorre algum tipo de interação entre a molécula do hidrótropo e os tensoativos na forma micelar, pois nenhuma das curvas mostrou a transição relacionada com o ponto de agregação do NaPTS, sob estas condições. Porém, através da técnica DSC, não fica evidente se a agregação do NaPTS não ocorre ou é detectada juntamente com os processos de desidratação dos grupos $\mathrm{PO}$ e separação de fases das soluções dos copolímeros.

Adicionando-se NaPTS ao sistema e aumentando-se sua concentração, os valores de $\Delta \mathrm{H}$ dos processos de micelização e separação de fases diminuem. Isto sugere que, devido ao aumento de solubilidade provocado pelo NaPTS, está ocorrendo a formação de um menor número de micelas ou a formação de micelas com um menor número de moléculas de tensoativo.

Através dos resfriamentos, não foi possível obter alguma informação a respeito da agregação das misturas de $\mathrm{NaPTS} /$ copolímero, devido às condições de análises não mostrarem a agregação de NaPTS.

\section{Conclusão}

Apesar de não ser a técnica mais indicada para o estudo dos tensoativos em solução aquosa, apresentando certas limitações, todo o estudo calorimétrico mostrou resultados concordantes com técnicas convencionais.

Os resultados de ponto de turvação obtidos por DSC são concordantes com aqueles obtidos pelo método visual[ ${ }^{[19]}$. Isto é favorável, já que se pode estudar a separação de fases de soluções aquosas de tensoativos utilizando-se uma técnica que propicia menores erros e resultados mais confiáveis.

\section{Referências Bibliográficas}

1. Schmolka, I.R. - "Polyalkylene oxide block copolymers", in: Nonionic Surfactants, M.J. Schick; F.M. Fowkes (ed.), Marcel Dekker, Inc., New York, vol.1, p.: 300334 (1966)

2. Bailey, F.E. \& Koleske, J.V.- “Configuration and hidrodynamic properties of polyoxyethylene chain in solutions", in: Nonionic Surfactants, M.J. Schick; F.M. Fowkes (ed.), Marcel Dekker, Inc., New York, vol.2, p.: 794-5 (1966)
3. Saeki, S.; Kuwahara, N.; Nakata, M. \& Kanenko, M. Polymer, 17, p.685 (1976)

4. Tadros, T.F. - "Surfactants, Industrial Applications", in: Encyclopedia of Physical Science and Technology, R.A. Meyers (ed.), Academic Press Inc., Orlando, vol.10, p.: 462-464 (1987)

5. Gilbert, J.C.; Hadgraft, J.; Bye, A. \& Brookes, L.G. Int. J. Pharm., 32, p.223 (1986)

6. Miyazaki, S.; Yokouchi, C.; Nakamura, T.; Hashiguchi, N.; Hou, W. \& Takada, M. - Chem. Pharm.Bull., 34, p.1801 (1986)

7. Napper, D.H. - "Polymeric Stabilization of Colloidal Dispersions", Academic Press, New York, p.: 1-2 (1983)

8. Linse, P. \& Malmsten, M. - Macromolecules, 25, p.5434 (1992)

9. Malmsten, M. \& Lindman, B. - Macromolecules, 25, p.5440 (1992)

10. Mortensen, K. \& Pedersen, J.S. - Macromolecules, 26, p.805 (1993)

11. Brown, W.; Schillen, K.; Almgren, M.; Hvidt, S. \& Bahadur, P. - J.Phys.Chem., 95, p.1850 (1991)

12. Alexandridis, P.; Holzwarth, J.F. \& Hatton, T.A. Macromolecules, 27, p.2414 (1994)

13. Chu, B. - Langmuir, 11, p.414 (1995)

14. Nivaggioli, T.; Alexandridis, P. \& Hatton, T.A. Langmuir, 11, p.730 (1995)

15. Alexandridis, P.; Nivaggioli, T. \& Hatton, T.A. Langmuir, 11, p.1468 (1995)

16. Hurter, P.N.; Scheutjens, J.M.H.M. \& Hatton, T.A. Macromolecules, 26, p.5030 (1993)

17. Hurter, P.N.; Scheutjens, J.M.H.M. \& Hatton, T.A. Macromolecules, 26, p.5592 (1993)

18. Oliveira, C.M.F. \& Lucas, E.F. - Polymer Bulletin, 24, p.363 (1990)

19. Mansur, C.R.E.; Oliveira, C.M.F.; González, G. \& Lucas, E.F. - J.Appl.Polym.Sci., 66, p.1767 (1997)

20. Mansur, C.R.E.; Spinelli, L.S.; Oliveira, C.M.F.; González, G. \& Lucas, E.F., J.Appl.Polym.Sci., 69, p.2459 (1998)

21. Mansur, C.R.E.; Spinelli, L.S.; González, G. \& Lucas, E.F., Colloids and Interfaces. Part A, 1998, in press

22. Balasubramanian, D.; Srinivas, V.; Gaikar, V.G. \& Sharma, M.M. - J.Phys.Chem., 93, p.3865 (1989)

Recebido: 29/09/98 Aprovado: 06/04/99 